\title{
Feasibility of Automated Foundational Ontology Interchangeability
}

\author{
Zubeida Casmod Khan ${ }^{1,2}$ and C. Maria Keet $^{1}$ \\ 1 Department of Computer Science, University of Cape Town, South Africa \\ mkeet@cs.uct.ac.za \\ 2 Council for Scientific and Industrial Research, Pretoria, South Africa \\ zkhan@csir.co.za
}

\begin{abstract}
While a foundational ontology can solve interoperability issues among the domain ontologies aligned to it, multiple foundational ontologies have been developed. Thus, there are still interoperability issues among domain ontologies aligned to different foundational ontologies. Questions arise about the feasibility of linking one's ontology to multiple foundational ontologies to increase its potential for uptake. To answer this, we have developed the tool SUGOI, Software Used to Gain Ontology Interchangeability, which allows a user to interchange automatically a domain ontology among the DOLCE, BFO and GFO foundational ontologies. The success of swapping based on equivalence varies by source ontology, ranging from 2 to $82 \%$ and averaging at $36 \%$ for the ontologies included in the evaluation. This is due to differences in coverage, notably DOLCE's qualities and BFO and GFO's roles, and amount of mappings. SUGOI therefore also uses subsumption mappings so that every domain ontology can be interchanged, preserves the structure of the ontology, and increases its potential for usability.
\end{abstract}

\section{Introduction}

The growth in the amount of Semantic Web applications and ontology-mediated interoperability of complex software applications pushes demands for infrastructure to facilitate with semantic interoperability. Already from the early days of the Semantic Web, foundational ontologies have been proposed as a component to facilitate such interoperability, for they provide common high-level categories so that domain ontologies linked to them are also interoperable [13]. Over the past 15 years, multiple foundational ontologies have been developed, however, such as DOLCE, BFO [13], GFO [5], SUMO [15], and YAMATO [14]. This introduced the issue of semantic conflicts for domain ontologies that are linked to different foundational ontologies, if those foundational ontologies are indeed really different, and new questions for ontology engineers, chiefly being:

1. Which foundational ontology should one choose to link one's domain ontology $O_{A}$ to?

2. If $O_{A}$ is linked to foundational ontology $O_{X}$, then is it still interoperable with domain ontology $O_{B}$ that is linked to foundational ontology $O_{Y}$ ? 
3. Is it feasible to automatically generate links between $O_{A}$ and $O_{Y}$ (which one may not know in sufficient detail), given $O_{A}$ is linked to $O_{X}$ ?

4. If there are issues with the former, what is causing it? Or: in praxis, which entities of $O_{X}$ are typically used for mappings with domain ontologies that may not be present, or present in an incompatible way, in $O_{Y}$ ?

The first question has been answered with ONSET [10], which considered the requirements but did not take into account what has been used in praxis. That is, ONSET cannot be used to answer the fourth question, which, may indicate actual modelling motivations and content of $O_{A}$ to chose $O_{X}$ over $O_{Y}$, assuming $O_{X}$ has those things being represented in $O_{A}$ that $O_{Y}$ does not have. If $O_{Y}$ has all those things as well, then there must be another reason why $O_{X}$ was chosen.

The aim of this paper is to answer questions 3 and 4, above. To achieve this, we designed and implemented SUGOI, a Software Used to Gain Ontology Interchangeability, which automatically interchanges the foundational ontology a domain ontology is linked to, as, to the best of our knowledge, no such tool exists yet. The current version can interchange between DOLCE, BFO, and GFO, for their mappings have been studied in detail $[8,11]$, but the system is designed for extensibility so as to handle any 'swap' (only new mapping files have to be provided). SUGOI is available from the foundational ontology library ROMULUS at http://www.thezfiles.co.za/ROMULUS/ontologyInterchange.html. We conducted an evaluation with 16 ontologies, using both quantitative and qualitative means. The overall 'raw interchangeability' based solely on the equivalence mappings among the foundational ontologies, is $36.18 \%$ on average, ranging from $2.04 \%$ to $81.81 \%$, depending mainly on the source ontology. If one permits subsumption mappings for the interchange, then one's foundational ontology can be fully swapped for another. The main reasons for any 'low' interchange is a combination of limited foundational ontology mappings that maintain consistency of the resultant ontology, and coverage of the foundational ontology, in particular when that is used in the domain ontology to foundational ontology alignment.

The remainder of the paper is structured as follows. The design of SUGOI is described in Section 2, which is followed by the experimental evaluation in Section 3. A discussion is presented in Section 4 and conclusions in Section 5.

\section{Design of SUGOI}

SUGOI has been designed to interchange domain ontologies between DOLCE, BFO, and GFO by using OWL ontology mapping files. For the foundational ontology mediation, we will use the results obtained by $[8,11]$ : its equivalence and subsumption mappings between entities in the three different ontologies have been investigated in detail, are logically consistent, and are available as machine-processable OWL files from the ontology repository ROMULUS [9].

Because several ontology files are being used in the interchangeability, we describe here the terms used for each one:

- The Source Ontology $\left({ }^{s} \mathcal{O}\right)$ that the user wants to interchange, which comprises the Source Domain Ontology $\left({ }^{s} \mathcal{O}_{d}\right)$, with the domain knowledge component of the source ontology, the Source Foundational Ontology $\left({ }^{s} \mathcal{O}_{f}\right)$ that 
is the foundational ontology component of the source ontology that is to be interchanged, and any equivalence or subsumption mappings between entities in ${ }^{s} \mathcal{O}_{d}$ and ${ }^{s} \mathcal{O}_{f}$.

- The Target Ontology $\left({ }^{t} \mathcal{O}\right)$ is the ontology that has been interchanged, which comprises the Target Domain Ontology $\left({ }^{t} \mathcal{O}_{d}\right)$, with the domain knowledge component of the target ontology, and the Target Foundational Ontology $\left({ }^{t} \mathcal{O}_{f}\right)$ that is the foundational ontology that the user has selected to interchange to, and any equivalence or subsumption mappings between entities in ${ }^{t} \mathcal{O}_{d}$ and ${ }^{t} \mathcal{O}_{f}$.

- Mapping ontology: the mapping ontology between the ${ }^{s} \mathcal{O}_{f}$ and the ${ }^{t} \mathcal{O}_{f}$.

- Domain entity: an entity from ${ }^{s} \mathcal{O}_{d}$ or ${ }^{t} \mathcal{O}_{d}$.

The algorithm and its implementation are presented in the next two subsections.

\subsection{Foundational Ontology Interchangeability Algorithm}

The general idea of the algorithm behind SUGOI is that it accepts a ${ }^{s} \mathcal{O}$ consisting of a ${ }^{s} \mathcal{O}_{d}$ linked to a ${ }^{s} \mathcal{O}_{f}$ (either DOLCE, BFO or GFO) and coverts it to a ${ }^{t} \mathcal{O}$ with a different ${ }^{t} \mathcal{O}_{f}$. For this, SUGOI must have access to all the foundational ontologies and the mapping ontologies. The ${ }^{s} \mathcal{O}$ is provided by the user. It does not matter whether the ${ }^{s} \mathcal{O}_{d}$ is linked to a foundational ontology by an import or a merge. SUGOI accesses the remainder of the ontologies either by loading the ontology from the online URI, or by loading it from an offline file, depending on the version in use (see below).

Twenty mapping files are pre-loaded into SUGOI, allowing the user to interchange between DOLCE, BFO and GFO modules bi-directionally. These mappings do not result in an inconsistency, because any alignment that did has been removed [8]. After the interchange process, all the domain entities from the ${ }^{s} \mathcal{O}_{d}$ are present in the ${ }^{t} \mathcal{O}_{d}$. SUGOI links domain entities from the ${ }^{s} \mathcal{O}_{d}$ to the ${ }^{t} \mathcal{O}_{f}$ as follows. SUGOI maps a domain entity's superentity in the ${ }^{s} \mathcal{O}_{f}$ to its corresponding superentity in the ${ }^{t} \mathcal{O}_{f}$ using the mapping ontology. This is illustrated in Fig 1 for the entity dmop:DataType from the DMOP ontology [7], changing the link from DOLCE to one in GFO, and this resulting axiom is called a $G T$, good target linking axiom. If the domain entity's superentity does not have a corresponding mapping entity, SUGOI then treats that superentity as a domain entity and looks for a corresponding mapping entity at a higher level up in the taxonomy. Thus, eventually, the domain entity from the ${ }^{s} \mathcal{O}_{d}$ is mapped with onthe-fly subsumption. This is displayed for interchanging the entity dmop:Strategy from the DMOP ontology in Fig 1, and this resulting axiom is called a BT, bad target linking axiom. If an entity is subsumed by owl:Thing or topObjectProperty in the ${ }^{s} \mathcal{O}$, then it is represented the same way in the ${ }^{t} \mathcal{O}$.

Algorithm 1 shows the procedure for changing a foundational ontology, which is illustrated afterwards with a practical example with the SAO ontology. Since the algorithm refers to independent ontology files, any changes in the foundational ontologies and mappings will not affect either the algorithm fundamentally or the software. Also, any implementation can be extended easily, as other foun- 

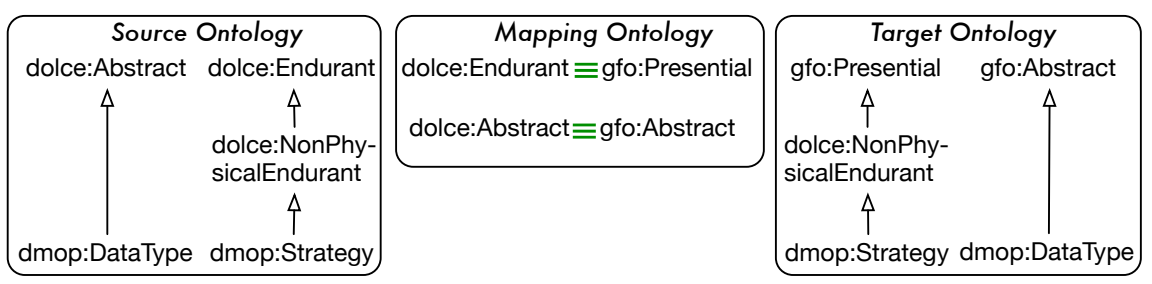

Fig. 1. Examples of interchanging domain entities dmop:DataType and dmop:Strategy from ${ }^{s} \mathcal{O}_{f}$ DOLCE to ${ }^{t} \mathcal{O}_{f}$ GFO with SUGOI, using equivalence and subsumption mappings, respectively.

dational ontologies and mappings are developed by including the new ontology file paths or URIs.

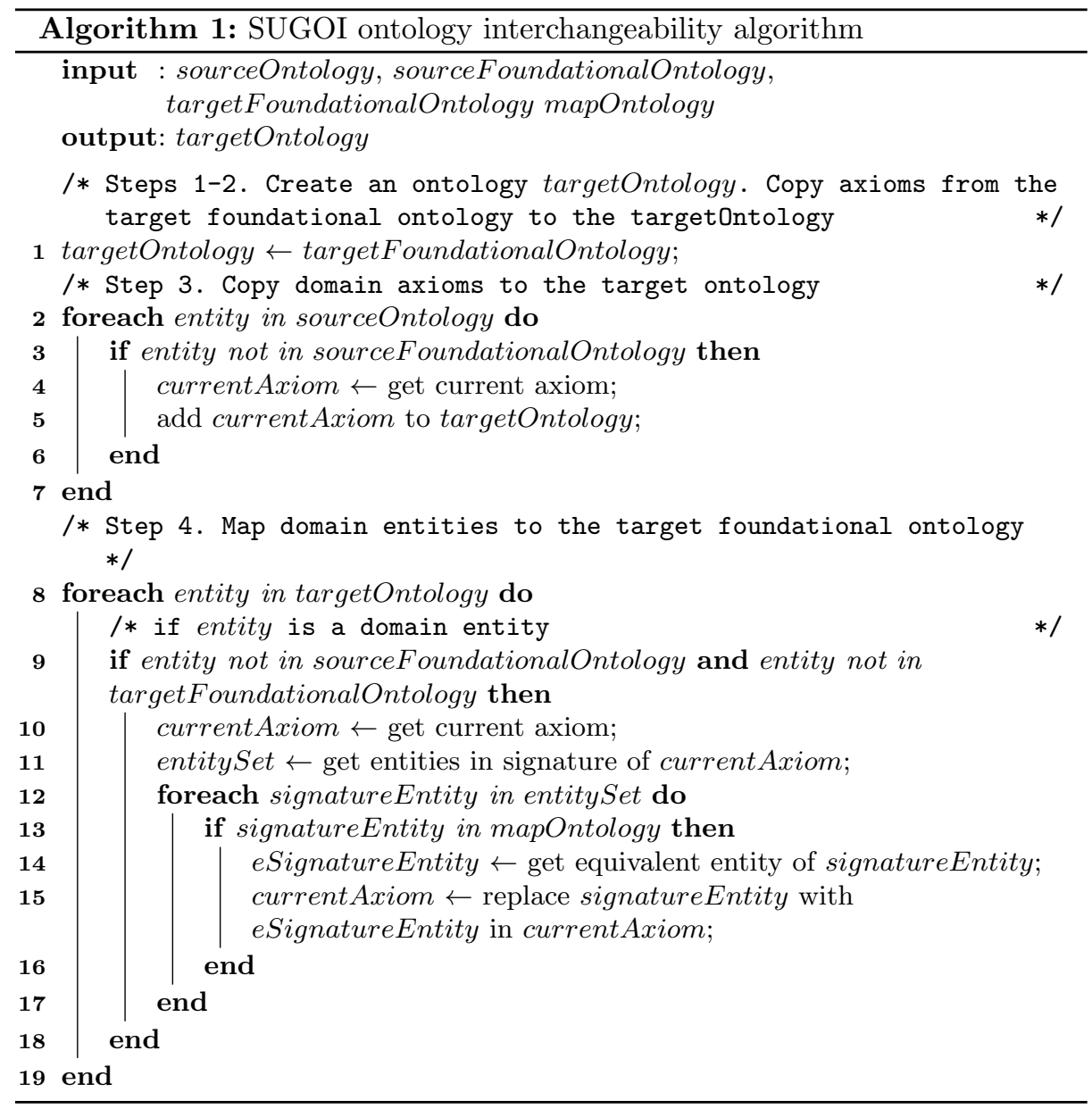




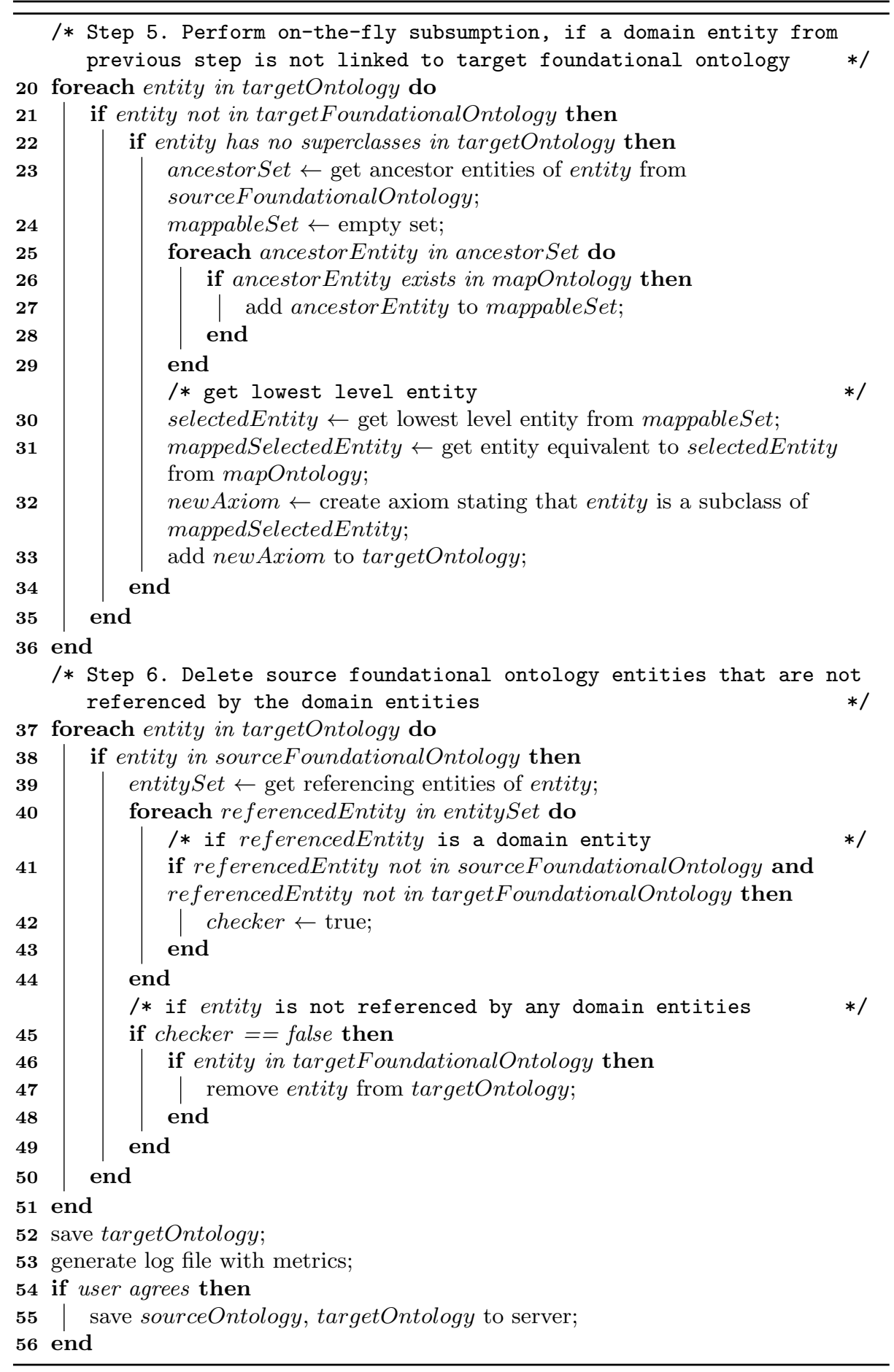


Example 1. The basic steps of the algorithm for interchanging between BFO to DOLCE are as follows, using the SAO ontology [12] as an example:

1. Create a new ontology file, a ${ }^{t} \mathcal{O}$ : sao-dolce.owl.

2. Copy the entire ${ }^{t} \mathcal{O}_{f}$ to the ${ }^{t} \mathcal{O}$ : copy the OWLized DOLCE ontology into sao-dolce.owl.

3. Copy the axioms from the ${ }^{s} \mathcal{O}_{d}$ to the ${ }^{t} \mathcal{O}$ : e.g., the axiom sao:Membrane Surface $\sqsubseteq$ bfo:Object_boundary exists in the ${ }^{s} \mathcal{O} \mathrm{SAO}$, which is added to the sao-dolce.owl ${ }^{t} \mathcal{O}$ and is referred to as a 'new' axiom.

4. Change the 'new' axioms to reference ${ }^{t} \mathcal{O}_{f}$ entities, if mappings exist: for the example in the previous step, no mapping exists for bfo:Object_boundary between BFO and DOLCE, so it proceeds to the next step.

5. If a mapping does not exist, perform on-the-fly subsumption: continuing with the example, bfo:Object_boundary has a superclass bfo:Independent_Continuant and the mapping ontology has bfo:Independent_Continuant $\equiv$ dolce:endurant, so bfo:Object_boundary $\sqsubseteq$ dolce:endurant is added to sao-dolce.owl.

6. Delete entities that exist in the ${ }^{t} \mathcal{O}$ that are from the ${ }^{s} \mathcal{O}_{f}$ but do not appear in an axiom with entities from the ${ }^{t} \mathcal{O}_{d}$, resulting in the final ${ }^{t} \mathcal{O}$, sao-dolce.owl.

\subsection{Implementation}

There are currently three platform-independent versions of SUGOI:

1. SUGOI applet: This version of SUGOI is an online web version and is integrated into the repository of foundational ontologies ROMULUS [9].

2. SUGOI desktop online version: This version is a platform independent jar file that is to be executed on a local machine, but requires internet connectivity.

3. SUGOI desktop offline version: This version is a platform independent jar file that is to be executed on a local machine, and is bundled together with dependent foundational and mapping ontology files.

SUGOI was developed in Java using the OWLAPI v3.5.0 in Netbeans IDE 8.0. The Applet of SUGOI is deployed online within any browser that has the Java TM Platform plugin installed and activated. The desktop versions of SUGOI are platform independent jar files together with their dependencies (all bundled together) that require minimal disk space, and Java runtime components installed. Fig. 2 is a screenshot of the online desktop version of SUGOI when interchanging an ontology from BFO to DOLCE.

\section{Experimental Evaluation}

The first purpose of the quantitative evaluation is to assess whether SUGOI successfully interchanges a ${ }^{s} \mathcal{O}$ to a ${ }^{t} \mathcal{O}$ and to determine the amount of the ontology that will be effectively interchanged, which refers to those entities within the ${ }^{t} \mathcal{O}$ that have been mapped with equivalence relations, thereby not required to use parts of the ${ }^{s} \mathcal{O}_{f}$ in the ${ }^{t} \mathcal{O}$. Second, to carry out a qualitative assessment of the entities and axioms to uncover what contributes to (un)successful interchangeability. Finally, although assessing whether the interchange matches some 'gold 


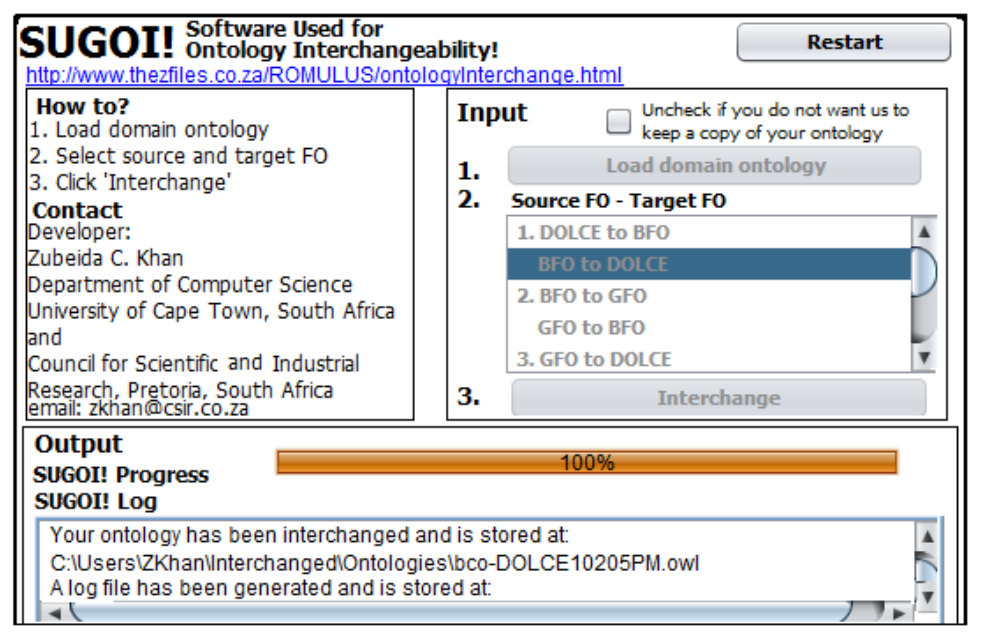

Fig. 2. The interface of the online desktop version of SUGOI.

standard' of dual manual mappings is unrealistic (there are too few ontologies that are linked to more than one foundational ontology), we consider two to cross-check whether there are any major differences between the manual and automated mappings obtained.

\subsection{Materials and methods}

Materials The SUGOI desktop application online version was used for the automated interchangeability, Protégé v4.3 for data on ontology changes, and a set of real ontologies to evaluate. Based on the ${ }^{s} \mathcal{O}_{f}$ of the ontologies, we load five out of the twenty mapping files of SUGOI, for interchanging between DOLCE $\leftrightarrow$ BFO, $\mathrm{BFO} \leftrightarrow \mathrm{GFO}, \mathrm{GFO} \leftrightarrow \mathrm{DOLCE}, \mathrm{DOLCE} \leftrightarrow \mathrm{GFOBasic}$, and GFOBasic $\leftrightarrow \mathrm{BFO}$.

The sample size was 16 source ontologies covering various subject domains, of which 5 have DOLCE as ${ }^{s} \mathcal{O}_{f}, 6$ with BFO, and 3 with GFO. For DOLCE we select the following ontologies: DMOP.owl, which is an ontology about data mining optimisation [7], the Naive_animal_ontology2.owl ontology of animals ${ }^{3}$, OntoDerm_5.3.owl about dermatology [4], the SceneOntology.owl ontology of spatial scenes and objects specifically for visual recognition ${ }^{4}$, and SEGOv3.owl for describing relations between geographic occurrences and properties observed by sensors $^{5}$. For BFO, we select the following ontologies: bco.owl about biological collections ${ }^{6}$, the epidemiology_ontology.owl for the epidemiology and pub-

\footnotetext{
${ }^{3}$ http://di.unito.it/ radicion/datasets/aic_13/Naive_animal_ontology.owl

${ }^{4}$ http://www.informatik. uni-bremen.de/ joana/ontology/modSpace/ 
lic health domain ${ }^{7}$, ero.owl intended for representing biomedical research resources $^{8}$, ID0.9.19.07. owl representing infectious diseases [3], the proper_name_on -tology_v1.8. owl that contains proper names for re-use in biomedical ontologies $^{9}$, and the SAO. owl ontology about subcellular anatomy of the nervous system [12]. For GFO as ${ }^{s} \mathcal{O}_{f}$, we select the following ontologies: the pid.owl about primary immunodeficiency diseases [1] and two biological core ontologies, gfo-bio. owl, which is a biological ontology of individuals, and gfo-bio-meta.owl, which is a biological ontology of categories [6]. We could not find other OWL ontologies using GFO.

For the cross-check of SUGOI with existing source ontologies, we are aware of only BioTop [2] that has been linked manually to both BFO and DOLCE, and one by one of the authors, the Stuff ontology ${ }^{10}$ about macroscopic stuff (amounts of matter), which had been linked manually to DOLCE and to BFO in a different project. Although limited, it is useful to evaluate them with SUGOI and compare its output with the manual mappings.

All test files used for this evaluation can be downloaded from ROMULUS at http://www.thezfiles.co.za/ROMULUS/sugoitestfiles.zip.

Methodology The methodology for the experiment is as follows.

Preprocess domain ontologies: This involves checking whether each ${ }^{s} \mathcal{O}$ uses the latest version of its respective ${ }^{s} \mathcal{O}_{f}$ (DOLCE-Lite version 397, BFO version 1.1, and GFO version 1.0) and checking whether the ontology import URIs are correct. If the ${ }^{s} \mathcal{O}$ in question uses an older version of the foundational ontology or the import URIs do not work, we correct this.

Perform interchangeability: Run the SUGOI application for each ${ }^{s} \mathcal{O}$ twice to acquire its respective target ontologies; e.g., if the ${ }^{s} \mathcal{O}_{f}$ is GFO, we generate two versions for the ${ }^{t} \mathcal{O}$, one with DOLCE and another with BFO.

Evaluate interchangeability: We evaluate the target ontologies as follows:

1. Compare the metrics of the domain entities of the target ontologies to those of the ${ }^{s} \mathcal{O}$.

2. Compare the ${ }^{t} \mathcal{O}$ to the ${ }^{s} \mathcal{O}$ using the compare feature in Protégé. Protégé generates a list of entities that have been added, deleted and modified. Its 'modified entities' refers to the axioms that are used to define the entities, and whether they have been changed. In each ${ }^{t} \mathcal{O}$, the entities that are modified are those that reference the mappable classes from their respective foundational ontologies.

3. Running the reasoner for the ${ }^{t} \mathcal{O}$ to detect if there are unsatisfiable entities. 4. Analyse the metrics of the ${ }^{s} \mathcal{O}_{f}$ entities that exist in the target ontologies.

\footnotetext{
7 https://epidemiology-ontology.googlecode.com/files/epo.owl

8 http://eagle-i.googlecode.com/svn/releases/2013-08-02/ero.owl

${ }^{9}$ http://proper-name-ontology.googlecode.com/files/proper_name_ontology_ v1.8.ow1

${ }^{10}$ http://www.meteck.org/files/ontologies/stuff.owl
} 
5. Analyse the raw interchangeability of each ${ }^{t} \mathcal{O}$, i.e., the amount of the ${ }^{t} \mathcal{O}$ that has been correctly interchanged using equivalence mappings thereby not referring to the ${ }^{s} \mathcal{O}_{f}$ entities. This is calculated from the ${ }^{t} \mathcal{O}$ as follows: Let $G T$, good target linking axioms, represent the sum of axioms that link domain ontology entities and ${ }^{t} \mathcal{O}_{f}$ entities in the ${ }^{t} \mathcal{O}$. Let $B T$, bad target linking axioms, represent the sum of axioms that link domain ontology entities and ${ }^{s} \mathcal{O}_{f}$ entities in the ${ }^{t} \mathcal{O}$; the raw interchangeability is calculated as follows:

$$
\text { Raw interchangeability }=\frac{|G T|}{|G T+B T|} \times 100
$$

For instance, recall Fig. 1: the subsumption with dolce:NonPhysicalEndurant in the ${ }^{t} \mathcal{O}$ counts toward the bad target linking axioms, whereas dmop:DataType $\sqsubseteq$ gfo:abstract counts as a good target linking axiom. SUGOI generates the raw interchangeability and ontology metrics in its log file for each ${ }^{t} \mathcal{O}$.

6. Analyse and compare the DOLCE- and BFO-linked BioTop and Stuff ontologies with SUGOI's interchangeability. We interchange in both directions and compare the output with the original ontologies.

Because there are not many domain ontologies linked to a foundational ontology, there will be insufficient data to conduct a full statistical analysis to compare the results for different interchanges.

\subsection{Results and discussion}

Preprocessing the source ontologies ought not to be necessary, as they should use the latest versions of the foundational ontologies, and all imports should work. The DMOP ${ }^{s} \mathcal{O}$ had to be pre-processed as the URI of its imported ontology DMOPObsolete did not work. The $\mathrm{SAO}^{s} \mathcal{O}$ also had to be pre-processed as it referred to version 1.0 of the $\mathrm{BFO}$ ontology, which is not the current version.

Analysis of the interchanged ontologies Table 1 displays the domain entities in the ${ }^{s} \mathcal{O}_{d}$ of the ${ }^{s} \mathcal{O}$ and the domain entities of the ${ }^{t} \mathcal{O}_{d^{-}}$-component of the ${ }^{t} \mathcal{O}$ for the source ontologies. The 'modified entities' are those where a subsumption changed in the process; e.g., in the original DOLCE version of the DMOP ontology, the dmop:DecisionBoundary class is a subclass of dolce:abstract, which is modified in the GFO version, where dmop:DecisionBoundary is a subclass of gfo:Abstract. Such changes were collected from Protégé, which is illustrated for the example in Fig. 3.

Comparing the metrics of the domain entities of the ${ }^{s} \mathcal{O}_{d}$ and ${ }^{t} \mathcal{O}_{d}$ shows there are one or more extra domain entities in the ${ }^{t} \mathcal{O}_{d}$, indicating the amount of ${ }^{s} \mathcal{O}_{f}$ entities that have been added to the ${ }^{t} \mathcal{O}_{d}$ (recall Example 1 and Fig. 1). The number of domain ontology entities increases from ${ }^{s} \mathcal{O}_{d}$ to ${ }^{t} \mathcal{O}_{d}$ : e.g., $\delta=(749-$ $739)=7$ when interchanging DMOP to a GFO-aligned version (see Table 1), which is due to some absent mappings between the ${ }^{s} \mathcal{O}_{f}$ and ${ }^{t} \mathcal{O}_{f}$. The same occurs with the set of BFO-aligned and GFO-aligned ${ }^{s} \mathcal{O}$, and they differ for each case. Among others, bfo:Object_boundary is added to the ${ }^{t} \mathcal{O}$ when the SAO 
Table 1. Comparison of the ${ }^{s} \mathcal{O}_{d}$ entities to the ${ }^{t} \mathcal{O}_{d}$ entities and raw interchangeability; interchang. $=$ raw interchangeability and avg $=$ average.

\begin{tabular}{|c|c|c|c|c|c|c|}
\hline $\begin{array}{l}\text { Source and Target } \\
\text { ontology }\end{array}$ & 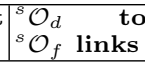 & $\begin{array}{l}\text { Domain } \\
\text { Classes }\end{array}$ & \begin{tabular}{|l} 
Domain \\
OP
\end{tabular} & $\begin{array}{l}\text { Domain } \\
\text { Entities }\end{array}$ & $\begin{array}{l}\text { Modified } \\
\text { entities }\end{array}$ & $\begin{array}{l}\text { Raw inter- } \\
\text { changeability }\end{array}$ \\
\hline \multicolumn{7}{|c|}{ Source domain ontologies linked to DOLCE with target domain ontologies } \\
\hline dmop & 409 & 739 & 140 & 1350 & & \\
\hline dmop-bfo & & 749 & 154 & 1377 & 43 & $4.88 \%$ \\
\hline dmop-gfo & & 745 & 154 & 1373 & 54 & $11.65 \%$ \\
\hline naive_animal & 41 & 416 & 15 & 438 & & \\
\hline naive_animal-bfo & & 422 & 24 & 453 & 20 & $21.95 \%$ \\
\hline naive_animal-gfo & & 421 & 24 & 452 & 22 & $25.58 \%$ \\
\hline ontoderm & 14 & 239 & 28 & 301 & & \\
\hline ontoderm-bfo & & 244 & 30 & 308 & 47 & $28.57 \%$ \\
\hline ontoderm-gfo & & 243 & 30 & 307 & 49 & $42.85 \%$ \\
\hline scene & 18 & 172 & 74 & 246 & & \\
\hline scene-bfo & & 175 & 78 & 253 & 3 & $50.00 \%$ \\
\hline scene-gfo & & 174 & 78 & 252 & 4 & $50.00 \%$ \\
\hline sego & 43 & 75 & 43 & 139 & & \\
\hline sego-bfo & & 89 & 55 & 165 & 32 & $29.54 \%$ \\
\hline sego-gfo & & 88 & 53 & 162 & 32 & $36.36 \%$ \\
\hline \multicolumn{3}{|c|}{ Correlation ${ }^{s} \mathcal{O}_{d^{-}}{ }^{s} \mathcal{O}_{f}$ links and interchang.: } & -0.79 & Avg for $L$ & $O L C E:$ & $30.02 \%$ \\
\hline
\end{tabular}

\begin{tabular}{|c|c|c|c|c|c|c|}
\hline \multicolumn{7}{|c|}{ Source domain ontologies linked to BFO with target domain ontologies } \\
\hline bco & 26 & 63 & 62 & 146 & & \\
\hline bco-dolce & & 67 & 62 & 150 & 19 & $65.39 \%$ \\
\hline bco-gfo & & 66 & 62 & 149 & 20 & $67.86 \%$ \\
\hline epidemiology & 15 & 169 & 4 & 173 & & \\
\hline epidemiology-dolce & & 174 & 4 & 178 & 13 & $60.00 \%$ \\
\hline epidemiology-gfo & & 173 & 4 & 177 & 15 & $68.75 \%$ \\
\hline ero & 97 & 3910 & 123 & 4114 & & \\
\hline ero-dolce & & 3918 & 123 & 4122 & 13 & $32.99 \%$ \\
\hline ero-gfo & & 3917 & 123 & 4121 & 51 & $52.85 \%$ \\
\hline ido & 77 & 150 & 0 & 150 & & \\
\hline ido-dolce & & 151 & 0 & 151 & 64 & $81.81 \%$ \\
\hline ido-gfo & & 151 & 0 & 151 & 65 & $81.81 \%$ \\
\hline proper_name & 13 & 30 & 34 & 64 & & \\
\hline proper_name-dolce & & 33 & 34 & 67 & 31 & $61.53 \%$ \\
\hline proper_name-gfo & & 32 & 34 & 66 & 26 & $76.92 \%$ \\
\hline sao & 54 & 728 & 36 & 809 & & \\
\hline sao-dolce & & 732 & 36 & 813 & 29 & $50.00 \%$ \\
\hline sao-gfo & & 731 & 36 & 812 & 29 & $55.17 \%$ \\
\hline \multicolumn{3}{|c|}{ Correlation ${ }^{s} \mathcal{O}_{d-}{ }^{s} \mathcal{O}_{f}$ links and interchang.: } & -0.33 & \multicolumn{2}{|c|}{ Avg for BFO: } & $62.90 \%$ \\
\hline
\end{tabular}

\begin{tabular}{|c|c|c|c|c|c|c|}
\hline \multicolumn{7}{|c|}{ Source domain ontologies linked to GFO with target domain ontologies } \\
\hline pid & 98 & 135 & 2 & 137 & & \\
\hline pid-dolce & & 138 & 9 & 147 & 22 & $14.29 \%$ \\
\hline pid-bfo & & 139 & 9 & 148 & 4 & $2.04 \%$ \\
\hline gfo-bio & 70 & 90 & 6 & 96 & & \\
\hline gfo-bio-dolce & & 103 & 12 & 115 & 19 & $20.27 \%$ \\
\hline gfo-bio-bfo & & 103 & 13 & 116 & 19 & $17.56 \%$ \\
\hline gfo-bio-meta & 14 & 127 & 6 & 139 & & \\
\hline gfo-bio-meta-dolce & & 142 & 13 & 161 & 6 & $20 \%$ \\
\hline gfo-bio-meta-bfo & & 142 & 13 & 161 & 8 & $19.69 \%$ \\
\hline \multicolumn{3}{|c|}{ Correlation ${ }^{s} \mathcal{O}_{d}-{ }^{s} \mathcal{O}_{f}$ links and interchang.: } & -0.66 & \multirow{2}{*}{\multicolumn{2}{|c|}{$\begin{array}{l}\text { Avg for GFO: } \\
\text { Avg for all: }\end{array}$}} & $15.64 \%$ \\
\hline \multicolumn{4}{|c|}{ Correlation all ${ }^{s} \mathcal{O}_{d}-{ }^{s} \mathcal{O}_{f}$ links and interchang.: -0.44} & & & $36.18 \%$ \\
\hline
\end{tabular}

\begin{tabular}{|c|c|c|c|}
\hline \multirow{2}{*}{$\begin{array}{l}\text { Modified: } \\
\text { DecisionBoundary }\end{array}$} & Description & Baseline Axiom & New Axiom \\
\hline & Superclass changed & $\begin{array}{l}\text { DecisionBoudary } \\
\text { SubClassOf abstract }\end{array}$ & $\begin{array}{l}\text { DecisionBoundary } \\
\text { SubClassOf Abstract }\end{array}$ \\
\hline
\end{tabular}

Fig. 3. A change for the dmop:DecisionBoundary class when interchanged to a GFO ${ }^{t} \mathcal{O}$. 
ontology is interchanged from $\mathrm{BFO}$ to DOLCE, because there is no mapping from bfo:Object_boundary to a DOLCE entity, whereas in the interchange to GFO, bfo:object_boundary maps to gfo:Material_boundary, so sao:Membrane Surface becomes a subclass of gfo:Material_boundary.

The number of entities in each ${ }^{t} \mathcal{O}$ that are from the ${ }^{s} \mathcal{O}_{f}$ follows from the extra domain entities in Table 1. In terms of these metrics, ontologies with a small difference in ${ }^{s} \mathcal{O}_{d}$ and ${ }^{t} \mathcal{O}_{d}$ numbers, i.e., having a low number of ${ }^{s} \mathcal{O}_{f}$ entities, perform best because they only contain few entities that cannot be mapped with equivalence, while ontologies with a high number of ${ }^{s} \mathcal{O}_{f}$ entities perform worst because they contain many domain entities that cannot be mapped with equivalence. The IDO ontology has the least number of ${ }^{s} \mathcal{O}_{f}$ entities in its ${ }^{t} \mathcal{O}_{d}$ (only 1), thus it performs the best, whereas the gfo-bio-meta performs the worst with $15{ }^{s} \mathcal{O}_{f}$ entities in its ${ }^{t} \mathcal{O}_{d}$. The extra domain entities in a ${ }^{t} \mathcal{O}$ ontology cause an increase in the number of BT, bad target linking axioms, which causes a lower raw interchangeability. The raw interchangeability values for the ${ }^{t} \mathcal{O}$ files are shown in the last column of Table 1.

The interchanged ontologies are consistent, except for ido-dolce.owl and ido-gfo.owl, but ido.owl ${ }^{s} \mathcal{O}$ was already unsatisfiable due to conflicting disjointness and subclass axioms among some domain entities.

Entity-level analysis Let us now consider those extra domain entities, which are those that are commonly used in domain ontologies, but do not have corresponding equivalence mappings among the foundational ontologies; or: the main 'culprits' for a low interchangeability. Table 2 displays these results. For DOLCEaligned ${ }^{s} \mathcal{O}$ ontologies, the object property dolce:has-quality has been referenced the most at 308 times, followed by dolce:has-quale 180 times, which are used for relating an endurant (e.g., apple) to a property (e.g., colour) and a value (e.g., red). Hence, domain ontologies linked to DOLCE heavily use DOLCE's features for representing properties and values. While there is some support for representing properties and values in BFO and GFO, they are not represented in the same way. BFO does not have any object properties, so while properties are supported using the bfo:quality entity, there is no object property to link together an entity and its property. GFO does have a gfo:has_value and a gfo:value_of that correspond to those DOLCE entities 'in spirit', but this is not asserted in the corresponding mapping file due to conflicting domain and range axioms that would result in an unsatisfiable ontology. Other DOLCE entities that have been referenced many times include dolce:inherent-in, and dolce:abstract-region. For BFO-interchanged ontologies, the bfo:Role entity has been used the most, at 72 times; clearly the BFO domain ontologies use thematic roles and perhaps the results could be improved if we considered interchanging these ontologies using the FunctionalParticipation module of DOLCE that does cover roles. Other frequently used BFO entities include bfo:Continuant, and bfo:Site. It might appear that bfo:Continuant could simply be mapped to the dolce:Endurant and gfo:Presential. This is not the case, however, because bfo:Continuant subsumes bfo:quality, and dolce:quality is disjoint from dolce:Endurant so it would result in an inconsistency in the ${ }^{t} \mathcal{O}$ if we did. It causes other inconsistencies when bfo:Continuant is mapped to gfo:Presential. 
Table 2. The number of times (N) a source foundational ontology entity is referenced in target ontologies for the total set of interchanged ontologies.

\begin{tabular}{|l|l||l|l|l|l|}
\hline DOLCE entity & N & BFO entity & N & GFO entity & N \\
\hline has-quality & 308 & Role & 72 & plays_role & 80 \\
\hline has-quale & 180 & Continuant & 36 & part_of & 52 \\
\hline inherent-in & 88 & Site & 30 & has_participant & 47 \\
\hline abstract-region & 60 & Function & 19 & has_part & 34 \\
\hline non-physical-endurant & 28 & ProcessualEntity & 18 & has_property & 34 \\
\hline particular & 26 & ObjectAggregate & 17 & on_level & 32 \\
\hline non-physical-object & 20 & FiatObjectPart & 8 & played_by & 30 \\
\hline mediated-relation & 18 & RealizableEntity & 6 & Biological_level & 28 \\
\hline mediated-relation-i & 14 & $\begin{array}{l}\text { GenericallyDependent } \\
\text { Continuant }\end{array}$ & 6 & has_role & 20 \\
\hline part & 14 & Disposition & 4 & instance_of & 20 \\
\hline $\begin{array}{l}\text { other DOLCE entities } \\
\text { (aggregated) }\end{array}$ & 170 & $\begin{array}{l}\text { other BFO entities } \\
\text { aggregated) }\end{array}$ & 13 & $\begin{array}{l}\text { other GFO entities } \\
\text { (aggregated) }\end{array}$ & 124 \\
\hline
\end{tabular}

Recall that the raw interchangeability measures the amount of the domain entities that have been interchanged using equivalence mappings (Table 1). Given the minimal set of satisfiable equivalence mappings -7 for DOLCE to BFO, 10 for BFO to GFO, and 15 for GFO to DOLCE [8] - it is no surprise that the average raw interchangeability for the source ontologies is only $36.18 \%$. The set of BFO ontologies had the highest raw interchangeability $(62.90 \%)$, followed by DOLCE $(30.02 \%)$ and lastly GFO (15.64\%). BFO has the highest raw interchangeability probably because it is a bare taxonomy with no entity axioms (other than disjointness axioms) and no object properties. The entities of DOLCE and GFO have many axioms that cause dependencies between entities, therefore if a domain entity is related to a foundational ontology entity, other foundational ontology entities are also affected. In general, the raw interchangeability differs greatly for the target ontologies which is due to two counterweighting factors. First, the number of links between the ${ }^{s} \mathcal{O}_{d}$ and ${ }^{s} \mathcal{O}_{f}$ has a moderate negative correlation with the raw interchangeability for DOLCE and GFO; see Table 1. Thus, a larger number of links between ${ }^{s} \mathcal{O}_{d}$ and ${ }^{s} \mathcal{O}_{f}$ entities for DOLCE and GFO ontologies can cause a lower raw interchangeability values, whereas for the set of $\mathrm{BFO}^{s} \mathcal{O}_{f}$, the correlation is much weaker. Second, the raw interchangeability is slightly higher when there are more mappings between source and target foundational ontologies among the interchanged ones: there are more DOLCE to GFO mappings (15) than DOLCE to BFO mappings (7), and the average interchangeability for the test ontologies are $33.29 \%$ and $26.99 \%$, respectively. The same pattern exists for BFO to DOLCE vs BFO to GFO (58.62\% vs $67.23 \%)$ and for GFO to BFO vs GFO to DOLCE $(13.10 \%$ vs $18.19 \%$ ). This does not hold for their aggregates, though, where the effect is dampened due to the large variation in raw interchangeability.

Comparing SUGOI to manual mappings Lastly, we evaluate SUGOI's interchangeability with the BFO and DOLCE versions of BioTop and Stuff to compare the existing manual mappings with the automatically generated ones.

The ontologies were interchanged and the raw interchangeability measure, and other metrics for the ${ }^{t} \mathcal{O}$ ontologies are displayed in Fig 3, which are in 
Table 3. The BioTop and Stuff ${ }^{t} \mathcal{O}$ ontology metrics regarding interchangeability and change in cross comparison.

\begin{tabular}{|l|l|l|l|l|}
\hline $\begin{array}{l}\text { Target ontology }\left({ }^{t} \mathcal{O}\right), \text { with the last } \\
\text { component of the name the }{ }^{t} \mathcal{O}_{f}\end{array}$ & $\begin{array}{l}\text { Raw } \\
\text { interchangeability }\end{array}$ & $\begin{array}{l}\text { New } \\
\text { entities }\end{array}$ & $\begin{array}{l}\text { Modified } \\
\text { entities }\end{array}$ & $\begin{array}{l}\text { Additional } \\
\text { mappings }\end{array}$ \\
\hline biotop-bfo-ro-dolce.owl & $41.18 \%$ & 25 & 60 & 4 \\
\hline biotop-dolce-ro-bfo.owl & $27.59 \%$ & 67 & 54 & 6 \\
\hline stuff-bfo-dolce.owl & $80.00 \%$ & 12 & 17 & 1 \\
\hline stuff-dolcelite-bfo.owl & $45.45 \%$ & 8 & 14 & 3 \\
\hline
\end{tabular}

the same range as with the other ontologies (cf. Table 2). The interchangeability measure for the BioTop ontologies stems from the different coverage in the two foundational ontologies. For instance, in the original DOLCE-aligned version, biotop:physical boundary $\sqsubseteq$ dolce:feature, while in the original BFO-aligned version, it is not directly subsumed by a BFO entity. This also means that the manual versions of BioTop will differ from the interchanged versions. Comparing the interchanged versions of BioTop (e.g., biotop-bfo-ro-dolce.owl) to the original manual versions (e.g., biotop-dolce.owl), we note that there are some new and modified entities, and additional ${ }^{t} \mathcal{O}_{d}$ to ${ }^{t} \mathcal{O}_{f}$ subsumption axioms identified by SUGOI. One of the additional links in biotop-dolce-ro-bfo.owl is, biotop:ImmaterialObject $\sqsubseteq$ bfo:MaterialEntity, which is a consequence of biotop:ImmaterialObject $\sqsubseteq$ dolce-physical-endurant in the original biotop-dolce-ro.owl, and there is a new subsumption biotop:ValueRegion $\sqsubseteq$ dolce:endurant in biotop-bfo-ro-dolce . owl, which is also due to the ${ }^{s} \mathcal{O}$, for biotop:ValueRegion $\sqsubseteq$ bfo:IndependentContinuant was asserted in the original biotop-bfo-ro.owl.

For the cross comparison of the Stuff ontologies, there are new and modified entities, and additional mapping axioms in the Stuff ${ }^{t} \mathcal{O}$ ontologies. One of the additional links in stuff-dolcelite-bfo.owl is, stuff:Endurant $\equiv$ bfo:IndependentContinuant, while in stuff-bfo-dolce.owl, there is, stuff:Perdurant $\equiv$ dolce:process (a consequence of stuff:perdurant $\equiv$ bfo:process in the stuff-bfo.owl).

Overall, the Stuff ontology performed better in terms of raw interchangeability than the BioTop ontology, and compares well to the manual effort. However, the importance of using SUGOI for interchangeability in both ontologies is demonstrated by the fact that there were some missing mappings from the manual ontologies. Thus, it is best to use SUGOI in conjunction with manual interchangeability to ensure that all the relevant mappings have been implemented for the domain ontologies.

\section{Discussion}

Considering the results together, the average raw interchangeability for all the target ontologies is $36.18 \%$ (ranging between $2.04 \%$ to $81.81 \%$ ), which means there are typically more links thanks to subsumption rather than equivalence. This is due to the fact that the set of equivalence mappings among the foundational ontologies is limited, and in some cases, those non-mapped entities from the ${ }^{s} \mathcal{O}_{f}$ are heavily used in the alignment of the ${ }^{s} \mathcal{O}_{d}$ to the ${ }^{t} \mathcal{O}_{f}$, as seen by dolce:has-quality (Table 2). Foundational ontology developers may wish to add 
those entities to broaden the foundational ontology's coverage and therewith increase its interoperability. For the time being, it means that domain ontology developers should choose a foundational ontology carefully.

Interchangeability surely can be performed, and the subsumption mappings added by SUGOI improve the quality of the ${ }^{t} \mathcal{O}$ in that extra domain entities are subsumed by the relevant ${ }^{t} \mathcal{O}_{f}$ entities, resulting in a 'clean' taxonomy, i.e., entities that cannot be mapped via equivalence are not by default mapped as subclasses of owl:Thing or topObjectProperty outside the scope of the ${ }^{t} \mathcal{O}_{f}$.

The interchanged ontologies are indeed usable and SUGOI can be used as an initial tool used to achieve semantic interoperability with regards to foundational ontologies. The best results (higher raw interchangeability) were obtained for DOLCE ontologies when interchanging to GFO, for BFO ontologies when interchanging to GFO, and for GFO ontologies when interchanging to DOLCE.

We now return to the questions posed in the introduction. Regarding question 3: it is indeed feasible to automatically generate links between a domain ontology and a different foundational ontology, although the results based on equivalence-only mappings depend on the source ontology and its amount of links to its ${ }^{s} \mathcal{O}_{f}$. Permitting subsumption, then the whole ontology can be interchanged to another foundational ontology. Regarding question 4: the issues observed are due to a combination of varying foundational ontology coverage (notably quality properties and roles), the amount of mappings between foundational ontologies, and the amount of links between the domain and foundational ontology components of the source ontology.

\section{Conclusion}

We presented the design of the tool SUGOI, which automatically changes a source ontology's foundational ontology to another, maintaining alignments between the domain ontology component and the chosen foundational ontology (either DOLCE, BFO, or GFO). This automation enabled an investigation into the feasibility of aligning automatically one's ontology to another foundational ontology. The success of such a 'swap' based only on equivalence among entities in foundational ontologies differs by source ontology, ranging from 2 to $82 \%$ success, and averaging at $36 \%$ for the 16 ontologies included in the evaluation. Comparing SUGOI to manual dual mappings, it did outperform manual efforts, in the sense of having found additional alignments, but also missed a few, thus a final manual check is advisable. The large differences in interchangeability success are due mainly to differences in coverage of the foundational ontology the number of alignment axioms between the source domain and foundational ontology, and to a lesser extent also the amount of mappings between each pair of foundational ontologies. SUGOI also uses subsumption mappings so that every domain ontology can be interchanged, preserving the structure of the ontology. SUGOI thus can be deployed in ontology engineering, and facilitating interoperability.

For future work, we consider creating mappings between other foundational ontologies and the existing ontologies in SUGOI. The community could also 
assist with this by submitting mappings in ROMULUS's community page at http://www.thezfiles.co.za/ROMULUS/communitySubmission.html. We also plan to extend ONSET [10] with content coverage aspects.

\section{References}

1. Adams, N., Hoehndorf, R., Gkoutos, G.V., Hansen, G., Hennig, C.: PIDO: The primary immunodeficiency disease ontology. Bioinformatics 27(22), 3193-3199 (2011)

2. Beisswanger, E., Schulz, S., Stenzhorn, H., Hahn, U.: BioTop: An upper domain ontology for the life sciences - a description of its current structure, contents and interfaces to OBO ontologies. Applied Ontology 3(4), 205-212 (2008)

3. Cowell, L.G., Smith, B.: Infectious disease ontology. In: Sintchenko, V. (ed.) Infectious Disease Informatics, pp. 373-395. Springer New York (2010)

4. Eapen, B.R.: ONTODerm-a domain ontology for dermatology. Dermatol Online Journal 14(6), 16 (2008)

5. Herre, H.: General Formal Ontology (GFO): A foundational ontology for conceptual modelling. In: Theory and Applications of Ontology: Computer Applications, chap. 14, pp. 297-345. Springer, Heidelberg (2010)

6. Hoehndorf, R., Loebe, F., Poli, R., Herre, H., Kelso, J.: GFO-Bio: A biological core ontology. Applied Ontology 3(4), 219-227 (2008)

7. Keet, C.M., Lawrynowicz, A., d'Amato, C., Hilario, M.: Modeling Issues, Choices in the Data Mining OPtimization Ontology. In: Proceedings of the 10th International Workshop on OWL: Experiences and Directions (OWLED 2013). CEUR Workshop Proceedings, vol. 1080. CEUR-WS.org (2013), Montpellier, France, May 26-27

8. Khan, Z., Keet, C.M.: Addressing issues in foundational ontology mediation. In: 5th International Conference on Knowledge Engineering and Ontology Development (KEOD'13). pp. 5-16. SCITEPRESS - Science and Technology Publications (2013), Vilamoura, Portugal, 19-22 September

9. Khan, Z., Keet, C.M.: The foundational ontology library ROMULUS. In: 3rd International Conference on Model \& Data Engineering (MEDI'13). LNCS, vol. 8216, pp. 200-211. Springer (2013), September 25-27, Amantea, Calabria, Italy

10. Khan, Z., Keet, C.M.: ONSET: Automated foundational ontology selection and explanation. In: ten Teije, A., et al. (eds.) 18th International Conference on Knowledge Engineering and Knowledge Management (EKAW'12). LNAI, vol. 7603, pp. 237-251. Springer (2012), Oct 8-12, Galway, Ireland

11. Khan, Z., Keet, C.M.: Toward semantic interoperability with aligned foundational ontologies in ROMULUS. In: Seventh International Conference on Knowledge Capture (K-CAP'13). ACM proceedings (2013), 23-26 June, Banff, Canada. (poster/demo)

12. Larson, S.D., Fong, L.L., Gupta, A., Condit, C., Bug, W.J., Martone, M.E.: A formal ontology of subcellular neuroanatomy. Front Neuroinform 1, 3 (2007)

13. Masolo, C., Borgo, S., Gangemi, A., Guarino, N., Oltramari, A.: Ontology library. WonderWeb Deliverable D18 (ver. 1.0, 31-12-2003). (2003), http://wonderweb.semanticweb.org

14. Mizoguchi, R.: YAMATO: Yet Another More Advanced Top-level Ontology. In: Proceedings of the Sixth Australasian Ontology Workshop. pp. 1-16. Conferences in Research and Practice in Information (2010), Sydney : ACS

15. Niles, I., Pease, A.: Towards a standard upper ontology. In: Welty, C., Smith, B. (eds.) Proceedings of the 2nd International Conference on Formal Ontology in Information Systems (FOIS-2001) (2001), Ogunquit, Maine, October 17-19, 2001 